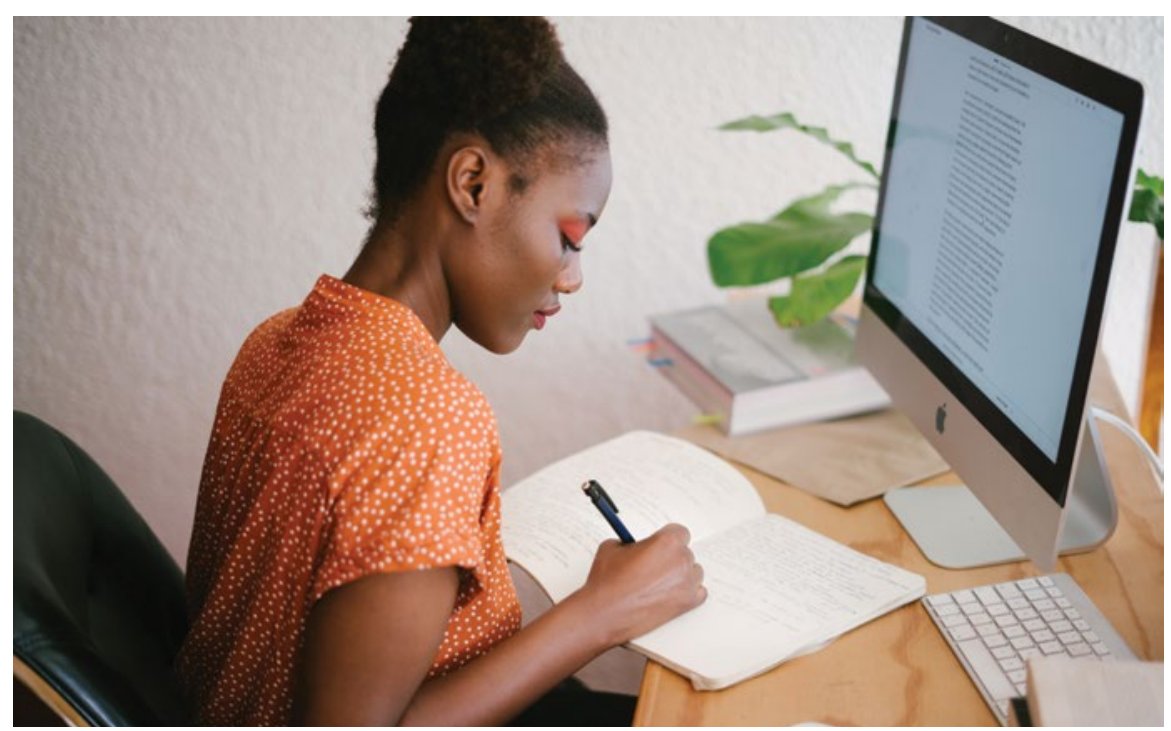

An innovative new accreditation scheme has just been launched to help dental practices reduce the risk of a GDC or CQC investigation by promoting their credibility, transparency, and reliability online.

The accreditation, known as The

Factuality Standard, was developed as a way for businesses, including dental practices, to prove the credibility and truthfulness of their online content, marketing material, and overall messaging whilst providing recognise honest and factual information.

Figures from the most recent GDC report covering 2018 reveal that 13 registrants had fitness to practise (FtP) cases brought against them for breaching GDC Guidance on Advertising. The report also revealed that $53 \%$ of FtP referrals were not generated by patient complaints but by other groups such as other dental professionals, the GDC itself, anonymously, or by the NHS or other public the public with a clear and simple way to bodies. Practices not complying with the GDC advertising guidance could find that an errant website is an easy target for an anonymous complaint by a disgruntled employee, patient or competitor practice.

By simply submitting their information for approval via the Factuality website, practices receive a detailed audit, recommendations, and certification once any suggested amendments needed to meet GDC and CQC guidance have been completed. They are then eligible for Factuality membership and may use the Factuality Trustmark across their publicity material.

The Factuality Standard is an instantly recognisable stamp of approval that demonstrates a practice's dedication to honesty, credibility and integrity, in accordance with the minimum standards required by enforcement authorities such as the GDC and CQC.

Trustmark holders are expected to gain a huge competitive advantage by quickly and easily demonstrating to patients and regulators that they are a trustworthy and reputable practice.

For more information about Factuality, visit: www.factuality.co.uk.

\title{
Facial visualisation tool launched
}

Align Technology, Inc. has announced the launch of its latest digital tool for general dentists and especially Invisalign Go Providers. The ClinCheck In-face Visualisation tool is an enhanced digital tool, which allows for an image of the patient's face to be incorporated into their treatment plan.

Actively engaging patients at every stage of their treatment is now simpler than ever before. By helping patients visualise their future smile, general dentists can clearly communicate the benefits of teeth straightening and guide patients towards the treatment choice most suitable for them.

Align Technology's latest enhancement to the 3D Clincheck software will take patient smile visualisation to the next level by helping dentists generate a powerful, clinical visualisation of the patient's teeth in their face. It will also enable general dentists to showcase benefits of teeth straightening and guide their patients

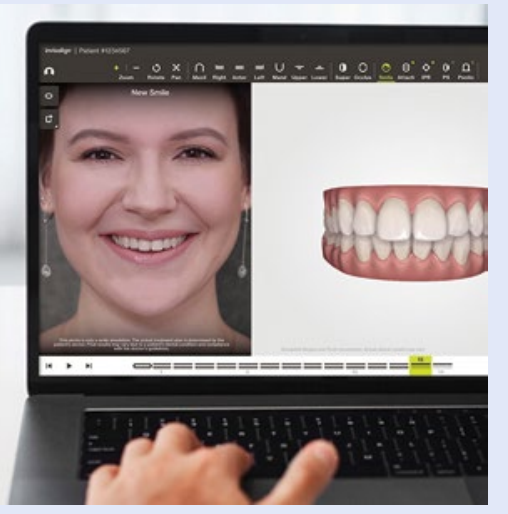

towards the most suitable treatment choices. This is made possible with the use of the Invisalign Photo Uploader application (available on iPhone and Android) to capture digital photos of the patient. The iTero intraoral scanner can then capture digital records in a more convenient and efficient way.

Align's proprietary ClinCheck software has long provided practitioners with 3D imaging of tooth movements from start to finish so they have options for the patient treatment journey. Now, with the added benefit of the 'In-face' Visualisation, ClinCheck allows dentists to customise treatment plans according to the patient's facial feature to make the visualisation more natural.

The ClinCheck 'In-face' Visualisation tool is now available for general practitioners using the Invisalign Go system in the United Kingdom and Ireland.

For more information, visit www. invisalign-go.co.uk. 